\title{
Supplement: A Decision-Theoretic Comparison of Treatments to Resolve Air Leaks After Lung Surgery Based on Nonparametric Modeling
}

\section{A. MCMC Implementation}

For reference, we restate the complete model. We implement inference under the DDP prior using the finite DP of Ishwaran and James (2001).

Sampling model: $Y_{j i} \mid \nu_{j 0}, M_{j} \sim \nu_{j 0} \delta_{0}+\left(1-\nu_{j 0}\right) M_{j}$ with

$$
M_{j}=\sum_{h=1}^{H} w_{h} N\left(Y_{j i} \mid \theta_{j h}, \sigma^{2}\right) \text { and } w_{h}=v_{h} \prod_{l<h}\left(1-v_{l}\right) .
$$

For posterior simulation, we replace the mixture in $M_{j}$ by an equivalent hierarchical model with latent indicator variables $\boldsymbol{r}=\left\{r_{j i}\right\}$

$$
\begin{aligned}
Y_{j i} \mid r_{j i}=h, Y_{j i} \neq 0 & \sim N\left(Y_{j i} \mid \theta_{j h}, \sigma^{2}\right) \\
p\left(r_{j i}=h\right) & =w_{h} .
\end{aligned}
$$

Note here that if $Y_{j i}=0, r_{j i}$ is not defined.

DDP prior: a (finite) DDP prior on $M_{0}, M_{1}$ is specified as

$$
\begin{aligned}
\boldsymbol{\theta}_{h} \mid \kappa, \tau= & \left(\theta_{0 h}, \theta_{1 h}\right) \sim M^{\star}, \\
& M^{\star}\left(\boldsymbol{\theta}_{h}\right)=N\left(\theta_{1 h} \mid \mu_{1}, \sigma_{1}^{2}\right)\left(\kappa I\left(\theta_{0 h}=\theta_{1 h}\right)+(1-\kappa) N_{+}\left(\theta_{0 h} \mid \theta_{1 h}, \tau^{2}\right)\right) . \\
v_{h} \mid \alpha \sim & \operatorname{Beta}(1, \alpha), h=1, \ldots, H-1 \text { and } v_{H}=1,
\end{aligned}
$$


Hyperpriors: Recall that we use the mapping $\zeta_{0}=\nu_{00}, \zeta_{1}=\nu_{10}-\nu_{00}$.

$$
\begin{gathered}
p\left(\zeta_{0}, \zeta_{1}\right)=\operatorname{Dir}(0.1,0.1,0.1), p\left(\sigma^{-2}\right)=\mathrm{Ga}(0.001,0.001), p\left(\tau^{-2}\right)=\mathrm{Ga}(0.5,0.5), \\
p(\kappa)=\operatorname{Beta}(1,1), \text { and } p(\alpha)=\mathrm{Ga}(1,1) .
\end{gathered}
$$

Assume that $N$ patients are randomized equally between the control and Progel group, that is $n_{0}+n_{1}=N$ and $n_{0}=n_{1}$. We implement posterior MCMC simulation using the following transition probabilities. The parameters are drawn from the corresponding complete conditional posterior probability distribution.

1. Update $\nu_{00}$ and $\nu_{10}$.

Sample $\left(\zeta_{0}, \zeta_{1}\right) \mid \ldots \sim \operatorname{Dir}\left(0.1+\lambda_{0}, 0.1+\lambda_{1}-\lambda_{0}, 0.1+N-\lambda_{0}-\lambda_{1}\right)$, where $\lambda_{0}=\sum_{i=1}^{n_{0}} I\left(Y_{0 i}=0\right)$ and $\lambda_{1}=\sum_{i=1}^{n_{1}} I\left(Y_{1 i}=0\right)$. Then let $\nu_{00}=\zeta_{0}$ and $\nu_{10}=\zeta_{0}+\zeta_{1}$.

2. Update $r_{j i}: p\left(r_{j i}=h \mid Y_{j i} \neq 0, \ldots\right) \propto w_{h} p\left(Y_{j i} \mid \theta_{j h}, \sigma^{2}\right), j=0,1 ; i=1, \ldots, n_{j}$.

3. Update $\sigma^{2}: \sigma^{2} \mid \ldots \sim \operatorname{InvGa}\left(0.5+\frac{N_{1}}{2}, 0.5+\frac{\sum_{h=1}^{H} \sum_{j=0}^{1} \sum_{r_{j i}=h, Y_{j i} \neq 0}\left(Y_{j i}-\theta_{j h}\right)^{2}}{2}\right)$, where $N_{1}=N-\sum_{j=0}^{1} \sum_{i=1}^{n_{j}} I\left(Y_{j i}=0\right)$.

4. Update $w_{h}$ : first sample $v_{h} \sim \operatorname{Beta}\left(1+\bar{n}_{h}, \alpha+\sum_{j>h} \bar{n}_{j}\right)$, where $\bar{n}_{h}=\sum_{j=0}^{1} \sum_{i=1}^{n_{j}} I\left(r_{j i}=\right.$ $h)$ is the total number of observations such at $r_{j i}=h$. Then $w_{h}=v_{h} \prod_{j>h}\left(1-v_{h}\right)$.

5. Update $\alpha$. Using data augmentation, first sample $m$ from the distribution $\operatorname{Beta}(\alpha+$ $\left.1, N_{1}\right)$. Then sample the new $\alpha$ from $\alpha \sim \gamma \mathrm{Ga}(1+H, 1-\log (m))+(1-\gamma) \mathrm{Ga}(H, 1-$ $\log (m))$ where $\frac{\gamma}{1-\gamma}=\frac{H}{N_{1}(1-\log (m))}$.

6. Update $\kappa: \kappa \mid \ldots \sim \operatorname{Beta}\left(1+\sum_{h=1}^{H} I\left(\theta_{0 h}=\theta_{1 h}\right), 1+\sum_{h=1}^{H} I\left(\theta_{0 h}>\theta_{1 h}\right)\right)$

7. Update $\tau^{2}: \tau^{2} \mid \ldots \sim \operatorname{InvGa}\left(0.5+\frac{\sum_{h=1}^{H} I\left(\theta_{0 h}>\theta_{1 h}\right)}{2}, 0.5+\frac{\sum_{h=1}^{H}\left(\theta_{0 h}-\theta_{1 h}\right)^{2}}{2}\right)$

8. Update $\boldsymbol{\theta}_{h}$. 
(a) Update $\theta_{1 h}: \theta_{1 h} \sim N\left(\mu_{1 h}, V_{1 h}\right)$, where $V_{1 h}^{-1}=1 / \sigma_{0}^{2}+\sum_{j=0}^{1} \sum_{i=1}^{n_{j}} I\left(r_{j i}=h\right) / \sigma^{2}$, and $\mu_{1 h}=V_{1 h}\left\{\mu_{1} / \sigma_{1}^{2}+1 / \sigma^{2}\left[\sum_{i: r_{1 i}=h} Y_{1 i}+\sum_{i: r_{0 i}=h}\left(Y_{0 i}-\left(\theta_{0 h}-\theta_{1 h}\right)\right)\right]\right\}$.

(b) Update $\theta_{0 h}: \theta_{0 h} \sim \pi_{h} \delta_{\theta_{1 h}}+\left(1-\pi_{h}\right) N_{+}\left(\mu_{0 h}, V_{0 h}\right)$, where $V_{0 h}^{-1}=1 / \tau^{2}+\sum_{i=1}^{n_{0}} I\left(r_{0 i}=h\right) / \sigma^{2}, \mu_{0 h}=\theta_{1 h}+V_{0 h}\left\{\sum_{i=1}^{n_{0}} I\left(r_{0 i}=h\right)\left(Y_{0 i}-\theta_{1 h}\right) / \sigma^{2}\right\}$, and $\pi_{h}=\nu_{00} /\left[\nu_{00}+\left(1-\nu_{00}\right) \frac{\sqrt{2}\left(1-\Phi\left(\theta_{1 h} ; \mu_{0 h}, V_{0 h}\right)\right)}{\sqrt{\left.\pi \tau^{2} N\left(\theta_{1 h} ; \mu_{0 h}, V_{0 h}\right)\right)}}\right]$. Here $\Phi(\cdot)$ stands for cumulative distribution function of standard normal distribution.

\section{B. Model Assessment Simulation}

We first carried out a preliminary simulation study to better understand the nature and accuracy of posterior inference under the proposed model for a reasonable sample size. Assume $N=100$ patients were randomized equally between the control and treatment group, that is, we restricted the randomization to obtain $n_{0}=n_{1}=50$ in each arm. For simplicity, we set $\nu_{00}=\nu_{10}=0$. The data, defined as $Y=\log (T+1)$ for $T=$ air leak length, were generated from the following mixture of two normals: $Y_{j i} \sim 0.8 N\left(1.5,0.3^{2}\right)+$ $0.2 N\left(3,0.3^{2}\right)$ for the control group $j=0$, and $Y_{j i} \sim 0.8 N\left(1,0.3^{2}\right)+0.2 N\left(2.5,0.3^{2}\right)$ for the treatment group, $j=1$. The simulated data mimic what might be observed in a real trial.

We simulated 100 hypothetical datasets and carried out posterior inference under the proposed method for each simulated dataset. The hyperprior parameters were determined as described earlier. The MCMC algorithm was implemented with an initial burn-in of 1000 iterations, and a total of 2000 iterations, saving every 10-th iteration. We evaluated convergence diagnostics using the $\mathrm{R}$ package coda, and found no evidence for lack of convergence.

Figure S1 shows the group-specific Bayesian nonparametric density estimates $E\left(G_{j}\right.$ $\boldsymbol{Y}$ ) for $j=0$ and 1 . The black curve represents the simulation truth, and the grey dotted lines represent estimates for each of the 100 simulated datasets. The estimates are distributed around the true density curve, showing no evidence of substantial bias. 
This simulation case was deliberately chosen to represent a modest sample size and possible differences between control group and treatment group that may be seen in an actual trial. The inference under the proposed method incorporating the stochastic ordering constraint performed well, indicating small bias even with moderate sample size.

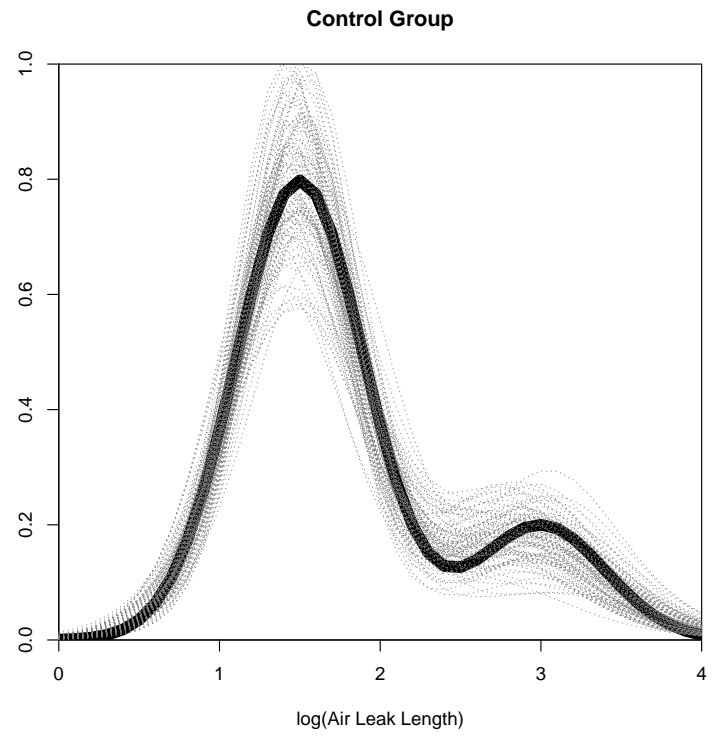

(a) $j=0$

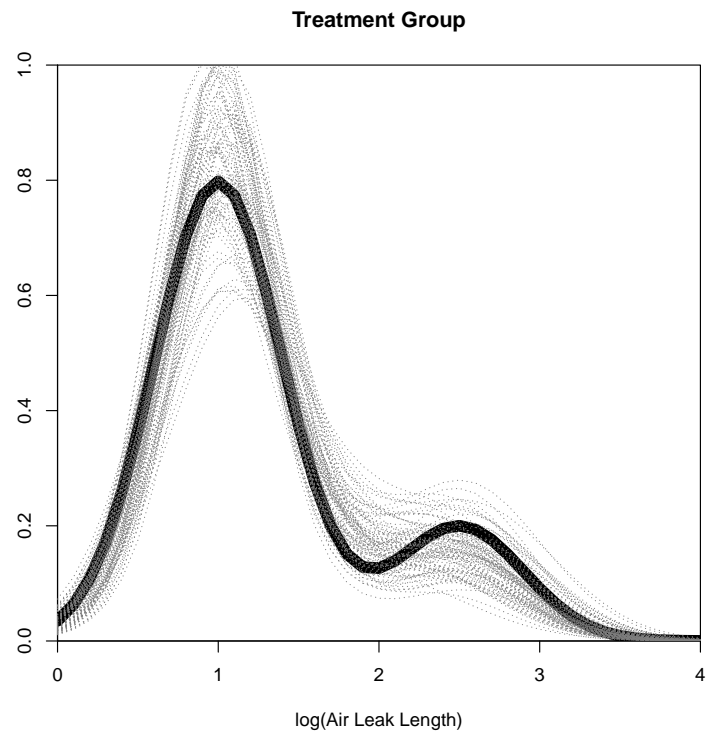

(b) $j=1$

Figure S1: Posterior density estimates $\bar{G}_{j}=E\left(G_{j} \mid \boldsymbol{Y}\right)$ for the control (a) and treatment groups (b), respectively. The thick black curves represent the simulation truth and the thin grey dotted lines are posterior estimates $\bar{G}_{j}$ for each of the 100 datasets.

\section{Simulation Results}

$\underline{\text { Scenarios } 1 \text { and } 2}$ are two null cases. In Scenario 1, the response outcomes $Y_{j i}$ in the control group and treatment group were generated by resampling from the historical trial data described in Figure 1. In Scenario 2, the response outcomes $Y_{j i}$ were generated by the same model $0.1 \delta_{0}+0.63 N\left(2, \bar{\sigma}^{2}\right)+0.27 N\left(3, \bar{\sigma}^{2}\right)$, where $\bar{\sigma}=0.3$. Both groups have the same mean utility 23.44. Thus, Scenarios 1 and 2 explored the ability of the proposed BNP design to stop if Progel performs no better than standard care. In Scenario 1, the average number of treated patients is 16.8 , which indicates that the trial is very likely to be stopped quickly after enrolling 16 patients. All of the 100 simulated trials are stopped 
early due to futility. The average number of patients treated in Scenario 2 is 28.8. Among 100 simulated trials, 64 are stopped early due to futility, and the type I error is 0.02 .

In $\underline{\text { Scenario } 3}$, the response outcomes in the control group were generated by a mixture of a point mass at 0 with probability 0.1 and three normal distributions with means 2.5 , 3, 4.5, respectively, and standard deviation 0.3 , while the response outcomes in the treatment group were generated by a mixture of point mass at 0 with probability 0.3 and three normal distributions with means 1, 2, 3.5, respectively, and standard deviation 0.3. The true mean utilities are $U_{1}^{o}=57.25, U_{0}^{o}=15.61$, and the difference is 41.64, which is much larger than $\epsilon_{U}=18$. Among the 100 trials simulated, 81 are stopped early due to superiority. In this scenario, the average number of patients enrolled is 29.12 and the PCD is 1.

In $\underline{\text { Scenario } 4}, Y_{1 i} \sim 0.2 \delta_{0}+0.56 N\left(1.5,0.3^{2}\right)+0.24 N\left(2,0.3^{2}\right)$, and $Y_{0 i} \sim 0.1 \delta_{0}+$ $0.63 N\left(1.8,0.3^{2}\right)+0.27 N\left(3,0.3^{2}\right)$. The true mean utilities are $U_{1}^{o}=48.94, U_{0}^{o}=29.79$, and the difference is 19.15, which is close to the target $\epsilon_{U}=18$. Among the 100 trials simulated, 15 are stopped early due to superiority and 48 declare Progel superiority without early stopping. The average number of patients recruited is 40.16 and the PCD is 0.63 .

In Scenarios 5 and 6 , the two treatment groups have different numbers of normal components. In $\underline{\text { Scenario } 5}$, the true utilities are $U_{1}^{o}=64.82, U_{0}^{o}=35.14$, and the difference is 29.68. As expected, 60 trials are stopped early due to superiority among 100 simulated trials. The average number of patients is 31.68 and the PCD is 0.93 . In $\underline{\text { Scenario } 6}$, the utility difference is 34.15. Among 100 simulated trials, 65 are stopped early due to superiority. The average number of patients is 29.92 and the PCD is 0.94 . Comparing Scenarios 5 and 6 indicates that with larger mean utility difference, fewer patients are recruited and the PCD becomes larger.

The last three scenarios explored more flexible choices of mixture distributions. We took exponential distribution and Weibull distribution into consideration as components 
Table S1: Frequentist operating characteristics of estimation bias and RMSE.

\begin{tabular}{ccc} 
Scenario & Bias & RMSE \\
\hline 1 & 1.68 & 3.94 \\
\hline 2 & 5.78 & 8.96 \\
\hline 3 & -4.16 & 13.11 \\
\hline 4 & 0.48 & 11.68 \\
\hline 5 & 1.88 & 14.01 \\
\hline 6 & 0.26 & 15.21 \\
\hline 7 & -5.87 & 16.14 \\
\hline 8 & 2.59 & 11.10 \\
\hline 9 & 3.39 & 14.54
\end{tabular}

of mixture. The operating characteristics are summarized in Table 3.

\section{Estimation Bias and Root Mean Squared Error (RMSE)}

Table S1 presents the operating characteristics for estimation bias and RMSE under each scenario.

\section{E. Sensitivity Analyses}

Utility elicitation. We first carry out a sensitivity analysis with respect to the elicited utilities $u(T)$. We consider two other surgeons. Hypothetical surgeon \#1 places much higher utilities on getting out of the hospital by day 10 and later, say

\begin{tabular}{c|ccccccccc}
\hline $\mathrm{T}$ (days) & 0 & 5 & 10 & 15 & 20 & 25 & 30 & 35 & $\geq 40$ \\
\hline Utility & 100 & 80 & 50 & 30 & 20 & 10 & 5 & 2 & 0 \\
\hline
\end{tabular}

Alternative Elicited utilities $u_{1}(T)$.

Hypothetical surgeon \#2 places higher utilities somewhere between RM and hypothetical surgeon \#1, making each numerical value midway between, 


\begin{tabular}{c|ccccccccc}
\hline $\mathrm{T}$ (days) & 0 & 5 & 10 & 15 & 20 & 25 & 30 & 35 & $\geq 40$ \\
\hline Utility & 100 & 65 & 30 & 18 & 10 & 6 & 4 & 2 & 0 \\
\hline
\end{tabular}

Alternative Elicited utilities $u_{2}(T)$.

Table S2: Sensitivity analysis to elicited utilities.

\begin{tabular}{ccccccccc} 
Scenario & Utility & True Diff & MSS & PCD & $\operatorname{Pr}($ EarS $)$ & $\operatorname{Pr}($ FinS $)$ & $\operatorname{Pr}(\operatorname{EarF})$ & $\operatorname{Pr}(\operatorname{FinF})$ \\
\hline \multirow{2}{*}{2} & U0 & 0 & 28.80 & 0.98 & 0.01 & 0.01 & 0.64 & 0.34 \\
& U1 & 0 & 29.12 & 1.00 & 0.00 & 0.00 & 0.72 & 0.28 \\
& U2 & 0 & 28.48 & 1.00 & 0.00 & 0.00 & 0.67 & 0.33 \\
\hline \multirow{3}{*}{3} & U0 & 41.64 & 29.12 & 1.00 & 0.81 & 0.18 & 0.01 & 0.00 \\
& U1 & 41.80 & 32.00 & 1.00 & 0.72 & 0.28 & 0.00 & 0.00 \\
& U2 & 42.14 & 30.88 & 0.99 & 0.75 & 0.24 & 0.01 & 0.00 \\
\hline \multirow{2}{*}{4} & U0 & 19.15 & 40.16 & 0.63 & 0.15 & 0.48 & 0.15 & 0.22 \\
& U1 & 20.26 & 41.76 & 0.64 & 0.12 & 0.52 & 0.12 & 0.24 \\
& U2 & 19.99 & 41.76 & 0.62 & 0.11 & 0.51 & 0.12 & 0.26 \\
\hline
\end{tabular}

Table S2 presents the operating characteristics under three different utility elicitations, including the utilities that are reported in the main paper as $u_{0}(T)$, and the two alternative elicitations as $u_{1}$ and $u_{2}$.

Decision boundaries. Finally, we carry out sensitivity analysis with respect to a change of the decision boundaries $\xi_{L}$ and $\xi_{U}$ for the continuation decision. Table S3 compares operating characteristics under three (arbitrarily chosen) scenarios under three different choices for these thresholds. In summary, the operating characteristics move slightly in the expected direction, including, for example increased probability (under repeat simulation) of early stopping for futility with increasing lower bound $\xi_{L}$, as expected. Overall, the reported summaries raise no concerns about unexpected sensitivities. 
Table S3: Sensitivity analysis of the proposed BNP design with respect to $\xi_{U}$ and $\xi_{L}$, under scenarios 2,3 , and $4(S 2, S 3, S 4)$. MSS denotes the mean sample size of patients treated. TIE denotes type I error. PCD denotes the probability of making the correct decision. $\operatorname{Pr}($ EarS $)$ denotes the probability of stopping early due to superiority. $\operatorname{Pr}($ EarF $)$ denotes the probability of stopping early due to futility. $\operatorname{Pr}($ FinS $)$ denotes the probability of declaring superiority in final analysis without early stopping. $\operatorname{Pr}($ FinF $)$ denotes the probability of declaring futility in final analysis without early stopping. All probabilities are with respect to repeated simulation.

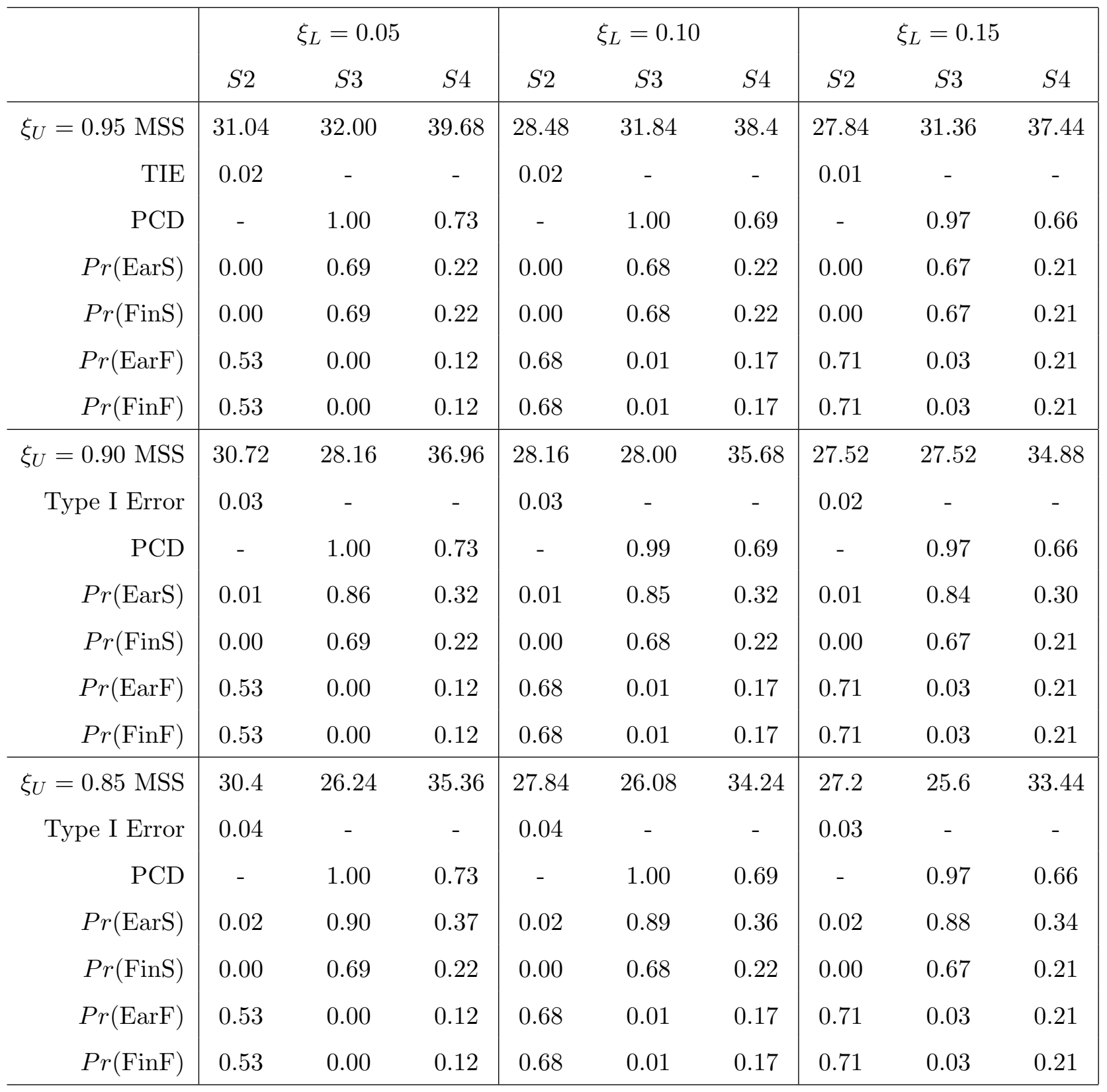




\section{References}

Ishwaran, H. and James, L. F. (2001). Gibbs sampling methods for stick-breaking priors.

Journal of the American Statistical Association, 96(453). 